This item was submitted to Loughborough's Research Repository by the author.

Items in Figshare are protected by copyright, with all rights reserved, unless otherwise indicated.

\title{
Hydrodynamic reductions of multidimensional dispersionless PDEs: The test
} for integrability

PLEASE CITE THE PUBLISHED VERSION

LICENCE

CC BY-NC-ND 4.0

REPOSITORY RECORD

Ferapontov, E.V., and Karima R. Khusnutdinova. 2019. "Hydrodynamic Reductions of Multidimensional Dispersionless Pdes: The Test for Integrability". figshare. https://hdl.handle.net/2134/299. 


\title{
Hydrodynamic reductions of multi-dimensional dispersionless PDEs: the test for integrability
}

\author{
E.V. Ferapontov ${ }^{*}$ and K.R. Khusnutdinova ${ }^{\dagger}$ \\ Department of Mathematical Sciences \\ Loughborough University \\ Loughborough, Leicestershire LE11 3TU \\ United Kingdom \\ e-mails: \\ E.V.Ferapontov@lboro.ac.uk \\ K. Khusnutdinova@lboro.ac.uk
}

\begin{abstract}
A $(d+1)$-dimensional dispersionless $\mathrm{PDE}$ is said to be integrable if its $n$ component hydrodynamic reductions are locally parametrized by $(d-1) n$ arbitrary functions of one variable. Given a PDE which does not pass the integrability test, the method of hydrodynamic reductions allows one to effectively reconstruct additional differential constraints which, when added to the equation, make it an integrable system in fewer dimensions (if consistent).
\end{abstract}

MSC: 35L40, 35L65, 37K10.

Keywords: Multi-dimensional Dispersionless Systems, Hydrodynamic Reductions, Integrability.

${ }^{*}$ Center for Nonlinear Studies, Landau Institute for Theoretical Physics, Kosygina 2, Moscow, 117940, Russia.

$\dagger$ On the leave from: Institute of Mechanics, Ufa Branch of the Russian Academy of Sciences, Karl Marx Str. 6, Ufa, 450000, Russia. 


\section{Introduction}

We address the problem of integrability of multi-dimensional dispersionless PDEs of the form

$$
F\left(u, u_{i}, u_{i j}\right)=0
$$

where $u$ is a (vector-) function of $d+1$ independent variables. For definiteness, let us consider $(3+1)$-dimesional PDEs in four independent variables $t, x, y, z$. Equations of this type naturally arise in mechanics, mathematical physics, general relativity and differential geometry. Let us look for exact solutions of (1) of the form $u=u\left(R^{1}, \ldots, R^{n}\right)$ where the Riemann invariants $R^{1}, \ldots, R^{n}$ solve a triple of commuting diagonal systems

$$
R_{t}^{i}=\lambda^{i}(R) R_{x}^{i}, \quad R_{y}^{i}=\mu^{i}(R) R_{x}^{i}, \quad R_{z}^{i}=\eta^{i}(R) R_{x}^{i} .
$$

Notice that the number of Riemann invariants is allowed to be arbitrary! Thus, the original multi-dimensional equation (1) is decoupled into a collection of commuting $(1+1)$ dimensional systems in Riemann invariants. In some cases one first needs to rewrite the original PDE (1) in a quasilinear form to make the method work: see examples in Sect.2. Solutions of this type, known as nonlinear interactions of $n$ planar simple waves, were investigated in gas dynamics and magnetohydrodynamics $[4,29]$. Later, they appeared in the context of the dispersionless KP hierarchy $[14,15,16,18,24,25,3]$. We will call a multi-dimensional equation integrable if it possesses 'sufficiently many' $n$-component reductions of the form (2) for arbitrary $n$ (the precise definition follows). Exact solutions arising within this approach can be viewed as dispersionless analogues of $n$-gap solutions.

Partial classification results of $(2+1)$-dimensional integrable systems of hydrodynamic type (the integrability is understood in the above sense) were obtained in our recent publications $[12,13]$. Integrable second order PDEs of the form $u_{t t}=f\left(u_{x x}, u_{x t}, u_{x y}\right)$ were classified in [28]. Particularly interesting examples arise in the theory of self-dual and Einstein-Weyl spaces $[30,8,9,10,11]$, in the context of the Dirichlet boundary problem in multi-connected domains [22], and the Whitham averaging procedure (in particular, the dispersionless limit) applied to $(2+1)$-dimensional solitonic PDEs [20, 21, 33].

We recall, see [32], that the requirement of commutativity of the flows (2) is equivalent to the following restrictions on their characteristic speeds:

$$
\frac{\partial_{j} \lambda^{i}}{\lambda^{j}-\lambda^{i}}=\frac{\partial_{j} \mu^{i}}{\mu^{j}-\mu^{i}}=\frac{\partial_{j} \eta^{i}}{\eta^{j}-\eta^{i}}
$$

$i \neq j, \partial_{j}=\partial / \partial_{R^{j}}$; no summation! Once these conditions are met, the general solution of (2) is given by the implicit 'generalized hodograph' formula [32]

$$
v^{i}(R)=x+\lambda^{i}(R) t+\mu^{i}(R) y+\eta^{i}(R) z, \quad i=1, \ldots, n,
$$

where $v^{i}(R)$ are characteristic speeds of the general flow commuting with (2), that is, the general solution of the linear system

$$
\frac{\partial_{j} v^{i}}{v^{j}-v^{i}}=\frac{\partial_{j} \lambda^{i}}{\lambda^{j}-\lambda^{i}}=\frac{\partial_{j} \mu^{i}}{\mu^{j}-\mu^{i}}=\frac{\partial_{j} \eta^{i}}{\eta^{j}-\eta^{i}} .
$$


Substituting $u\left(R^{1}, \ldots, R^{n}\right)$ into (1) and using (2) one arrives at an over-determined system for $\lambda^{i}(R), \mu^{i}(R), \eta^{i}(R)$ and $u(R)$ as functions of the Riemann invariants $R^{i}$. This system implies, in particular, that the characteristic speeds $\lambda^{i}, \mu^{i}$ and $\eta^{i}$ satisfy an algebraic relation which can be interpreted as the dispersion relation for the system (1).

One can show that the requirement of existence of nontrivial 3-component reductions is already sufficiently restrictive implying, in particular, the existence of $n$-component reductions for arbitrary $n$ [12]. This phenomenon is similar to the well-known three-soliton condition in the Hirota bilinear approach [19] (recall that two-soliton solutions exist for arbitrary PDEs transformable to Hirota's bilinear form and, therefore, cannot detect the integrability), and the condition of three-dimensional consistency in the classification of discrete integrable systems on quad-graphs [1]. One can show that the maximum number of $n$-component reductions a $(\mathrm{d}+1)$-dimensional PDE may possess is parametrized, modulo changes of variables $R^{i} \rightarrow f^{i}\left(R^{i}\right)$, by $(d-1) n$ arbitrary functions of one variable. Therefore, we propose the following

Definition. $A(d+1)$-dimensional PDE is said to be integrable if its $n$-component reductions are locally parametrized by $(d-1) n$ arbitrary functions of one variable.

One of the most important examples of PDEs in four dimensions which are integrable in this sense is the 'second heavenly' equation,

$$
\theta_{t x}+\theta_{z y}+\theta_{x x} \theta_{y y}-\theta_{x y}^{2}=0
$$

descriptive of self-dual Einstein spaces [30] (see Example 2 of Sect.2). Its equivalent 'first heavenly' form was discussed in the recent publication [11] where it was shown that $n$ component reductions are parametrized by $2 n$ arbitrary functions of a single variable. It would be interesting to compare these reductions with the solitonic reductions of [7]. The requirement of existence of $n$-component reductions parametrized by $2 n$ arbitrary functions of one variable appears to be very strong, indeed, the heavenly equation is the only nonlinear PDE of the form

$$
\theta_{t x}+\theta_{z y}=f\left(\theta_{x x}, \theta_{x y}, \theta_{y y}\right)
$$

which passes the integrability test (see the Appendix).

An interesting six-dimensional integrable generalization of the heavenly equation,

$$
\theta_{t \tilde{t}}+\theta_{z \tilde{z}}+\theta_{t x} \theta_{z y}-\theta_{t y} \theta_{z x}=0,
$$

arises in the context of $\operatorname{sdiff} f\left(\Sigma^{2}\right)$ self-dual Yang-Mills equations [31]. Its $n$-component reductions are parametrized by $4 n$ arbitrary functions of a single variable (Example 5 of Sect.3). We would like to thank M. Dunajski for drawing our attention to this equation.

Another integrable example is the six-dimensional system

$$
m_{t}=n_{x}+n m_{r}-m n_{r}, \quad n_{z}=m_{y}+m n_{s}-n m_{s},
$$

see Sect.3. Its $n$-component reductions depend on $4 n$ arbitrary functions of one variable. Under the additional constraints $m_{r}=n_{r}=0, s=x, z=t$, this system is descriptive of hyperCR Einstein-Weyl structures [6]; it was investigated in a series of recent publications $[27,9,26]$. 
In the Example 3 of Sect.2 we apply our method to the four-dimensional PDE

$$
F_{x z}=F_{x x} F_{x y}+F_{y t}
$$

which is the second flow of the dispersionless KP (dKP) hierarchy

$$
\begin{aligned}
& F_{x t}=\frac{1}{2} F_{x x}^{2}+F_{y y}, \\
& F_{x z}=F_{x x} F_{x y}+F_{y t},
\end{aligned}
$$

see, e. g., [5]. It is demonstrated that, considered separately, this equation is not integrable (as a four-dimensional PDE), the fact which is not at all surprising. What is more important, the method of hydrodynamic reductions allows one to effectively reconstruct the differential constraint $F_{x t}=\frac{1}{2} F_{x x}^{2}+F_{y y}$ which, when added to the equation, generates the $(2+1)$-dimensional dKP hierarchy.

Although the method of hydrodynamic reductions provides an infinity of (implicit) solutions parametrised by arbitrarily many functions of one variable, the question of solving the initial value problem for integrable multi-dimensional dispersionless PDEs remains open. A detailed investigation of the behavior and singularity structure of solutions describing nonlinear interactions of planar simple waves is beyond the scope of this paper.

\section{Examples}

In this section we list some examples of multi-dimensional PDEs which are integrable in the sense of hydrodynamic reductions.

Example 1. Let us consider the first two flows of the dispersionless KP hierarchy,

$$
\begin{gathered}
F_{x t}=\frac{1}{2} F_{x x}^{2}+F_{y y}, \\
F_{x z}=F_{x x} F_{x y}+F_{y t},
\end{gathered}
$$

which, in the new variables

$$
F_{x x}=u, F_{x y}=v, F_{y y}=w, F_{y t}=s, F_{x t}=\frac{1}{2} u^{2}+w, F_{x z}=u v+s,
$$

assume the quasilinear form

$$
\begin{gathered}
u_{y}=v_{x}, \quad v_{y}=w_{x}, \quad v_{t}=s_{x}, \quad w_{t}=s_{y}, \quad u_{t}=\left(\frac{1}{2} u^{2}+w\right)_{x}, \quad u_{z}=(u v+s)_{x}, \\
v_{t}=\left(\frac{1}{2} u^{2}+w\right)_{y}, \quad v_{z}=(u v+s)_{y}, \quad s_{x}=\left(\frac{1}{2} u^{2}+w\right)_{y}, \quad\left(\frac{1}{2} u^{2}+w\right)_{z}=(u v+s)_{t} .
\end{gathered}
$$

Notice that $u$ satisfies the dispersionless KP equation,

$$
\left(u_{t}-u u_{x}\right)_{x}=u_{y y}
$$


Looking for reductions in the form $u=u\left(R^{1}, \ldots, R^{n}\right), v=v\left(R^{1}, \ldots, R^{n}\right), w=w\left(R^{1}, \ldots, R^{n}\right)$, $s=s\left(R^{1}, \ldots, R^{n}\right)$ where the Riemann invariants $R^{i}$ satisfy (2), and substituting into (7), one readily obtains

$$
\partial_{i} v=\mu^{i} \partial_{i} u, \quad \partial_{i} s=\lambda^{i} \mu^{i} \partial_{i} u, \quad \partial_{i} w=\left(\mu^{i}\right)^{2} \partial_{i} u,
$$

along with the dispersion relations

$$
\lambda^{i}=u+\left(\mu^{i}\right)^{2}, \quad \eta^{i}=v+2 u \mu^{i}+\left(\mu^{i}\right)^{3} .
$$

The compatibility condition $\partial_{i} \partial_{j} v=\partial_{j} \partial_{i} v$ implies

$$
\partial_{i} \partial_{j} u=\frac{\partial_{j} \mu^{i}}{\mu^{j}-\mu^{i}} \partial_{i} u+\frac{\partial_{i} \mu^{j}}{\mu^{i}-\mu^{j}} \partial_{j} u,
$$

while the commutativity condition (3) results in

$$
\partial_{j} \mu^{i}=\frac{\partial_{j} u}{\mu^{j}-\mu^{i}} .
$$

The substitution of (11) into (10) implies the Gibbons-Tsarev system for $u(R)$ and $\mu^{i}(R)$,

$$
\partial_{j} \mu^{i}=\frac{\partial_{j} u}{\mu^{j}-\mu^{i}}, \quad \partial_{i} \partial_{j} u=2 \frac{\partial_{i} u \partial_{j} u}{\left(\mu^{j}-\mu^{i}\right)^{2}},
$$

$i \neq j$, which was first derived in $[15,16]$ in the context of hydrodynamic reductions of Benney's moment equations. For any solution $\mu^{i}, u$ of the system (12) one can reconstruct $\lambda^{i}, \eta^{i}$ and $v, s, w$ by virtue of the relations (9) and (10), which are automatically consistent. The general solution of the system (12) depends, modulo reparametrizations $R^{i} \rightarrow f^{i}\left(R^{i}\right)$, on $n$ arbitrary functions of one variable, thus manifesting the fact that PDEs (6) constitute a $(2+1)$-dimensional integrable system.

Example 2. The so-called second heavenly equation,

$$
\theta_{t x}+\theta_{z y}+\theta_{x x} \theta_{y y}-\theta_{x y}^{2}=0,
$$

is descriptive of self-dual Ricci-flat metrics [30]. Introducing the variables $\theta_{x x}=u, \theta_{x y}=v$, $\theta_{y y}=w, \theta_{t x}=p, \theta_{z y}=v^{2}-u w-p$, one can rewrite (13) in a quasilinear form,

$$
\begin{gathered}
u_{y}=v_{x}, \quad u_{t}=p_{x}, \quad v_{y}=w_{x}, \quad v_{t}=p_{y}, \\
v_{z}=\left(v^{2}-u w-p\right)_{x}, \quad w_{z}=\left(v^{2}-u w-p\right)_{y} .
\end{gathered}
$$

Hydrodynamic reductions are sought in the form $u=u\left(R^{1}, \ldots, R^{n}\right), v=v\left(R^{1}, \ldots, R^{n}\right)$, $w=w\left(R^{1}, \ldots, R^{n}\right), p=p\left(R^{1}, \ldots, R^{n}\right)$ where the Riemann invariants $R^{1}, \ldots, R^{n}$ solve a triple of commuting hydrodynamic type systems (2). The substitution into (14) implies

$$
\partial_{i} p=\lambda^{i} \partial_{i} u, \quad \partial_{i} v=\mu^{i} \partial_{i} u, \quad \partial_{i} w=\left(\mu^{i}\right)^{2} \partial_{i} u,
$$


along with the dispersion relation

$$
\lambda^{i}=2 v \mu^{i}-w-u\left(\mu^{i}\right)^{2}-\mu^{i} \eta^{i} .
$$

Substituting $\lambda^{i}$ into the commutativity conditions (3), and taking into account that the compatibility conditions for the relations $\partial_{i} p=\lambda^{i} \partial_{i} u, \partial_{i} v=\mu^{i} \partial_{i} u$ imply

$$
\partial_{i} \partial_{j} u=\frac{\partial_{j} \mu^{i}}{\mu^{j}-\mu^{i}} \partial_{i} u+\frac{\partial_{i} \mu^{j}}{\mu^{i}-\mu^{j}} \partial_{j} u
$$

one arrives at the following system:

$$
\begin{gathered}
\partial_{j} \mu^{i}=\frac{\left(\mu^{j}-\mu^{i}\right)^{2}}{\eta^{j}-\eta^{i}+u\left(\mu^{j}-\mu^{i}\right)} \partial_{j} u, \quad \partial_{j} \eta^{i}=\frac{\left(\mu^{j}-\mu^{i}\right)\left(\eta^{j}-\eta^{i}\right)}{\eta^{j}-\eta^{i}+u\left(\mu^{j}-\mu^{i}\right)} \partial_{j} u \\
\partial_{i} \partial_{j} u=2 \frac{\mu^{j}-\mu^{i}}{\eta^{j}-\eta^{i}+u\left(\mu^{j}-\mu^{i}\right)} \partial_{i} u \partial_{j} u .
\end{gathered}
$$

Solving equations (17) for $\mu^{i}, \eta^{i}$ and $u$, determining $\lambda^{i}$ from (16) and calculating $p, v, w$ from equations (15) (which are automatically compatible by virtue of (17)), one obtains the general $n$-component hydrodynamic reduction of the heavenly equation. Moreover, the commutativity conditions will also be satisfied identically.

We emphasize that the system (17) is in involution and its general solution depends on $3 n$ arbitrary functions of one variable. Indeed, one can arbitrarily prescribe the restrictions of $\mu^{i}$ and $\eta^{i}$ to the $R^{i}$-coordinate line. This gives $2 n$ arbitrary functions. Moreover, one can arbitrarily prescribe the restriction of $u$ to each of the coordinate lines, which provides extra $n$ arbitrary functions. However, since reparametrizations $R^{i} \rightarrow f^{i}\left(R^{i}\right)$ leave the system (17) invariant, one concludes that general n-component reductions are locally parametrized by $2 n$ arbitrary functions of one variable. This supports the evidence that the heavenly equation (13) is a true four-dimensional integrable PDE.

Obviously, the same method applies to other equivalent forms of the heavenly equation (13). For instance, hydrodynamic reductions of the first heavenly equation

$$
\Omega_{x y} \Omega_{z t}-\Omega_{x t} \Omega_{z y}=1
$$

were investigated in detail in [11]. Another possibility is to work with the evolutionary form [17] of the heavenly equation,

$$
\psi_{t t}=\psi_{x y} \psi_{z t}-\psi_{x t} \psi_{z y}
$$

In both cases one can derive analogues of equations (17) which, although involutive, look somewhat more complicated.

The requirement of existence of $n$-component reductions (parametrized by $2 n$ arbitrary functions of one variable) is very strong indeed: as demonstrated in the Appendix, the heavenly equation (13) is the only nonlinear PDE of the form

$$
\theta_{t x}+\theta_{z y}+f\left(\theta_{x x}, \theta_{x y}, \theta_{y y}\right)=0
$$

which passes the integrability test. 
Remark. The heavenly equation (and equivalent forms thereof) belong to the class of special Monge-Ampére equations which can be defined as follows. Consider a function $u\left(x^{1}, \ldots x^{k}\right)$ and introduce a $k \times k$ symmetric matrix $U=\left|u_{i j}\right|$ of its second partial derivatives. A special Monge-Ampere equation with constant coefficients is a PDE of the form

$$
M_{0}+M_{1}+\ldots+M_{k}=0
$$

where $M_{l}$ is a constant-coefficient linear combination of all distinct $l \times l$ minors of $U$, $0 \leq l \leq k$. Here, for instance, $M_{0}$ is a constant, $M_{k}=\operatorname{det} U=H e s s$, etc. Equivalently, this PDE can be obtained by equating to zero a constant-coefficient $k$-form in $2 k$ variables $x^{i}, u_{i}$. It is an interesting problem to classify integrable PDEs within this class, in particular, for $k=4$. We emphasize that the case $k=3$ is understood completely: one can show that, for $k=3$, any special Monge-Ampére equation is either linearizable by a contact transformation (in this case it is automatically integrable by the method of hydrodynamic reductions), or contact equivalent to either of the three nondegenerate forms $[23,2]$,

$$
\text { Hess } u=1, \quad \text { Hess } u=u_{11}+u_{22}+u_{33}, \quad \text { Hess } u=u_{11}+u_{22}-u_{33} \text {. }
$$

We have verified directly that these three PDEs are not integrable by the method of hydrodynamic reductions.

Example 3. Let us consider the second flow of the dispersionless KP hierarchy,

$$
F_{x z}=F_{x x} F_{x y}+F_{y t},
$$

see Example 1. The question is: should it be regarded as a four-dimensional integrable PDE? We will see that the answer to this question is negative, moreover, the method of hydrodynamic reductions applied to this equations reconstructs additional differential constraints which, when added to $(18)$, generate the $(2+1)$-dimensional dKP hierarchy. In the new variables

$$
F_{x x}=u, F_{x y}=v, F_{y y}=w, F_{y t}=s, F_{x t}=p, F_{x z}=u v+s,
$$

the equation (18) assumes the quasilinear form

$$
\begin{gathered}
u_{y}=v_{x}, \quad v_{y}=w_{x}, \quad v_{t}=s_{x}, \quad w_{t}=s_{y}, \quad u_{t}=p_{x}, \quad u_{z}=(u v+s)_{x}, \\
v_{t}=p_{y}, \quad v_{z}=(u v+s)_{y}, \quad s_{x}=p_{y}, \quad p_{z}=(u v+s)_{t} .
\end{gathered}
$$

Looking for reductions in the form $u=u\left(R^{1}, \ldots, R^{n}\right), v=v\left(R^{1}, \ldots, R^{n}\right), w=w\left(R^{1}, \ldots, R^{n}\right)$, $s=s\left(R^{1}, \ldots, R^{n}\right), p=p\left(R^{1}, \ldots, R^{n}\right)$, where the Riemann invariants $R^{i}$ satisfy $(2)$, and substituting into (19), one readily obtains

$$
\partial_{i} v=\mu^{i} \partial_{i} u, \quad \partial_{i} s=\lambda^{i} \mu^{i} \partial_{i} u, \quad \partial_{i} w=\left(\mu^{i}\right)^{2} \partial_{i} u, \quad \partial_{i} p=\lambda^{i} \partial_{i} u,
$$

along with the dispersion relation

$$
\eta^{i}=v+u \mu^{i}+\mu^{i} \lambda^{i}
$$


The compatibility condition $\partial_{i} \partial_{j} v=\partial_{j} \partial_{i} v$ implies

$$
\partial_{i} \partial_{j} u=\frac{\partial_{j} \mu^{i}}{\mu^{j}-\mu^{i}} \partial_{i} u+\frac{\partial_{i} \mu^{j}}{\mu^{i}-\mu^{j}} \partial_{j} u
$$

while the commutativity condition (3) results in

$$
\partial_{j} \mu^{i}=\frac{\mu^{j}+\mu^{i}}{\lambda^{j}-\lambda^{i}} \partial_{j} u, \quad \partial_{j} \lambda^{i}=\frac{\mu^{j}+\mu^{i}}{\mu^{j}-\mu^{i}} \partial_{j} u .
$$

The substitution of (23) into (22) implies the over-determined system for $\mu^{i}(R), \lambda^{i}(R)$ and $u(R)$,

$$
\begin{gathered}
\partial_{j} \mu^{i}=\frac{\mu^{j}+\mu^{i}}{\lambda^{j}-\lambda^{i}} \partial_{j} u, \quad \partial_{j} \lambda^{i}=\frac{\mu^{j}+\mu^{i}}{\mu^{j}-\mu^{i}} \partial_{j} u, \\
\partial_{i} \partial_{j} u=2 \frac{\mu^{j}+\mu^{i}}{\left(\mu^{j}-\mu^{i}\right)\left(\lambda^{j}-\lambda^{i}\right)} \partial_{i} u \partial_{j} u,
\end{gathered}
$$

$i \neq j$, which is analogous to the system (17). There is one crucial difference: the system $(24)$ is not in involution. Calculating compatibility conditions $\partial_{k}\left(\partial_{j} \mu^{i}\right)-\partial_{j}\left(\partial_{k} \mu^{i}\right)=0$, $\partial_{k}\left(\partial_{j} \lambda^{i}\right)-\partial_{j}\left(\partial_{k} \lambda^{i}\right)=0$ and $\partial_{k}\left(\partial_{j} \partial_{i} u\right)-\partial_{j}\left(\partial_{k} \partial_{i} u\right)=0$, one arrives at extra relations

$$
\begin{aligned}
& \left(\left(\mu^{i}\right)^{2}\left(\lambda^{k}-\lambda^{j}\right)+\left(\mu^{k}\right)^{2}\left(\lambda^{j}-\lambda^{i}\right)+\left(\mu^{j}\right)^{2}\left(\lambda^{i}-\lambda^{k}\right)\right) \times \\
& \left(\mu^{k} \mu^{j}\left(\lambda^{k}-\lambda^{j}\right)+\mu^{i} \mu^{j}\left(\lambda^{j}-\lambda^{i}\right)+\mu^{i} \mu^{k}\left(\lambda^{i}-\lambda^{k}\right)\right)=0 .
\end{aligned}
$$

Thus, there are two cases to consider.

Case 1. Equating to zero the first bracket in (25),

$$
\left(\mu^{i}\right)^{2}\left(\lambda^{k}-\lambda^{j}\right)+\left(\mu^{k}\right)^{2}\left(\lambda^{j}-\lambda^{i}\right)+\left(\mu^{j}\right)^{2}\left(\lambda^{i}-\lambda^{k}\right)=0,
$$

one obtains $\lambda^{i}=a\left(\mu^{i}\right)^{2}+b$. The substitution of this ansatz into (24) implies $\left(\mu^{i}\right)^{2} \partial_{j} a+$ $\partial_{j} b=\partial_{j} u$, so that $a=\alpha, b=u+\beta$ where $\alpha, \beta$ are arbitrary constants. Thus, $\lambda^{i}=$ $\alpha\left(\mu^{i}\right)^{2}+u+\beta$. Substituting $\lambda^{i}$ into the equation $\partial_{i} p=\lambda^{i} \partial_{i} u$ and using (20) one obtains, upon elementary integration, $p=\frac{1}{2} u^{2}+\beta u+\alpha w+\gamma$. Expressed in terms of second derivatives of $F$, this constraint reads

$$
F_{x t}=\frac{1}{2} F_{x x}^{2}+\beta F_{x x}+\alpha F_{y y}+\gamma
$$

One can show that the constants $\alpha, \beta, \gamma$ are not essentials and can be reduced to $\alpha=$ $1, \beta=\gamma=0$. Thus, the method of hydrodynamic reductions applied to the PDE (18) reconstructs the first flow of the dKP hierarchy, $F_{x t}=\frac{1}{2} F_{x x}^{2}+F_{y y}$.

Case 2. Equating to zero the second bracket in (25),

$$
\mu^{k} \mu^{j}\left(\lambda^{k}-\lambda^{j}\right)+\mu^{i} \mu^{j}\left(\lambda^{j}-\lambda^{i}\right)+\mu^{i} \mu^{k}\left(\lambda^{i}-\lambda^{k}\right)=0,
$$

one obtains $\lambda^{i}=\frac{a}{\mu^{i}}+b$. The substitution of this ansatz into (24) implies $\partial_{j} a+\mu^{j} \partial_{j} u+$ $\mu^{i}\left(\partial_{j} b+\partial_{j} u\right)=0$, so that $a=-v+\alpha, b=-u+\beta$ where $\alpha, \beta$ are arbitrary constants. Thus, $\lambda^{i}=\frac{\alpha-v}{\mu^{i}}+\beta-u$. Substituting $\lambda^{i}$ into the equation $\partial_{i} s=\lambda^{i} \mu^{i} \partial_{i} u$ and using (20) 
one has, upon elementary integration, $s=-u v+\alpha u+\beta v+\gamma$. Expressed in terms of second derivatives of $F$, this constraint reads

$$
F_{x x} F_{x y}+F_{y t}=\alpha F_{x x}+\beta F_{x y}+\gamma .
$$

Again, the constants $\alpha, \beta, \gamma$ are not essentials and can be reduced to zero. The resulting constraint $F_{y t}+F_{x x} F_{x y}=0$ characterizes stationary points of the flow (18). We have checked that this constraint is integrable in the sense of hydrodynamic reductions (as a $(2+1)$-dimensional PDE).

In any case, we conclude that the equation (18) is not integrable as a four-dimensional PDE. This is manifested by the fact that the system (24), which governs hydrodynamic reductions, is not in involution.

Example 4. Let us consider the system

$$
m_{t}=n_{x}, \quad n_{z}=m_{y}+m n_{x}-n m_{x}
$$

which, in the limit $z=t$, has been extensively investigated in $[27,9,26]$. Looking for reductions in the form $m=m\left(R^{1}, \ldots, R^{n}\right), n=n\left(R^{1}, \ldots, R^{n}\right)$ where the Riemann invariants $R^{i}$ satisfy (2), one obtains

$$
\partial_{i} n=\lambda^{i} \partial_{i} m, \quad \mu^{i}=\lambda^{i} \eta^{i}-m \lambda^{i}+n .
$$

The commutativity conditions (3) imply

$$
\partial_{j} \eta^{i}=\partial_{j} m, \quad \partial_{i} \partial_{j} m=0
$$

hence, up to reparametrizations $R^{i} \rightarrow \varphi^{i}\left(R^{i}\right)$, one has

$$
m=\sum_{k} R^{k}, \quad \eta^{i}=f^{i}\left(R^{i}\right)+\sum_{k} R^{k}
$$

where $f^{i}\left(R^{i}\right)$ are arbitrary functions of a single variable. The characteristic speeds $\lambda^{i}$ solve the linear system

$$
\frac{\partial_{j} \lambda^{i}}{\lambda^{j}-\lambda^{i}}=\frac{1}{f^{j}\left(R^{j}\right)-f^{i}\left(R^{i}\right)} .
$$

We refer to [27] for the general formula for $\lambda^{i}$ and further discussion of this example in the $(2+1)$-dimensional limit $z=t$. Thus, $n$-component hydrodynamic reductions of the system (26) are parametrized by $2 n$ arbitrary functions of one variable $\left(n\right.$ functions $f^{i}\left(R^{i}\right)$ plus $n$ functions in the general solution of the linear system for $\left.\lambda^{i}\right)$. Therefore, the fourdimensional system (26) is integrable. Notice that it can be obtained as the condition of commutativity of two vector fields,

$$
\left[\partial_{z}-m \partial_{x}-\lambda \partial_{x}, \quad \partial_{y}-n \partial_{x}-\lambda \partial_{t}\right]=0
$$

$\lambda=$ const, compare with [9]. Some further multi-dimensional generalizations of this example are discussed in Sect.3. 
Example 5. The six-dimensional generalization of the heavenly equation,

$$
\theta_{t \tilde{t}}+\theta_{z \tilde{z}}+\theta_{t x} \theta_{z y}-\theta_{t y} \theta_{z x}=0
$$

has been proposed in [31]. Introducing the variables $\theta_{t x}=a, \theta_{z y}=b, \theta_{t y}=p, \theta_{z x}=q$, $\theta_{z \tilde{z}}=r, \theta_{t \tilde{t}}=p q-a b-r$, one can rewrite (27) in a quasilinear form,

$$
\begin{gathered}
a_{y}=p_{x}, \quad a_{z}=q_{t}, \quad b_{t}=p_{z}, \quad b_{x}=q_{y}, \quad b_{\tilde{z}}=r_{y}, \quad q_{\tilde{z}}=r_{x}, \\
p_{\tilde{z}}=(p q-a b-r)_{y} .
\end{gathered}
$$

Hydrodynamic reductions are sought in the form $a=a\left(R^{1}, \ldots, R^{n}\right), b=b\left(R^{1}, \ldots, R^{n}\right)$, $p=p\left(R^{1}, \ldots, R^{n}\right), q=q\left(R^{1}, \ldots, R^{n}\right), r=r\left(R^{1}, \ldots, R^{n}\right)$, where the Riemann invariants $R^{1}, \ldots, R^{n}$ solve the commuting equations

$R_{x}^{i}=\lambda^{i}(R) R_{z}^{i}, \quad R_{y}^{i}=\mu^{i}(R) R_{z}^{i}, \quad R_{\tilde{z}}^{i}=\eta^{i}(R) R_{z}^{i}, \quad R_{t}^{i}=\beta^{i}(R) R_{z}^{i}, \quad R_{\tilde{t}}^{i}=\gamma^{i}(R) R_{z}^{i}$.

The substitution into (28) implies

$$
\partial_{i} p=\beta^{i} \partial_{i} b, \quad \partial_{i} r=\frac{\eta^{i}}{\mu^{i}} \partial_{i} b, \quad \partial_{i} q=\frac{\lambda^{i}}{\mu^{i}} \partial_{i} b, \quad \partial_{i} a=\frac{\lambda^{i} \beta^{i}}{\mu^{i}} \partial_{i} b,
$$

along with the dispersion relation

$$
\eta^{i}=\beta^{i} \mu^{i} q+\lambda^{i} p-\beta^{i} \lambda^{i} b-\mu^{i} a-\beta^{i} \gamma^{i}
$$

Substituting $\lambda^{i}$ into the commutativity conditions

$$
\frac{\partial_{j} \lambda^{i}}{\lambda^{j}-\lambda^{i}}=\frac{\partial_{j} \mu^{i}}{\mu^{j}-\mu^{i}}=\frac{\partial_{j} \eta^{i}}{\eta^{j}-\eta^{i}}=\frac{\partial_{j} \beta^{i}}{\beta^{j}-\beta^{i}}=\frac{\partial_{j} \gamma^{i}}{\gamma^{j}-\gamma^{i}}
$$

and taking into account that the compatibility conditions for the relations $\partial_{i} p=\beta^{i} \partial_{i} b$ imply

$$
\partial_{i} \partial_{j} b=\frac{\partial_{j} \beta^{i}}{\beta^{j}-\beta^{i}} \partial_{i} b+\frac{\partial_{i} \beta^{j}}{\beta^{i}-\beta^{j}} \partial_{j} b
$$

one arrives at the following system:

$$
\begin{gathered}
\frac{\partial_{j} \beta^{i}}{\beta^{j}-\beta^{i}}=\frac{\partial_{j} \lambda^{i}}{\lambda^{j}-\lambda^{i}}=\frac{\partial_{j} \mu^{i}}{\mu^{j}-\mu^{i}}=\frac{\partial_{j} \gamma^{i}}{\gamma^{j}-\gamma^{i}}=\frac{\lambda^{i}-\lambda^{j} \mu^{i} / \mu^{j}}{q\left(\mu^{j}-\mu^{i}\right)+b\left(\lambda^{i}-\lambda^{j}\right)+\gamma^{i}-\gamma^{j}} \partial_{j} b, \\
\partial_{i} \partial_{j} b=\frac{\lambda^{i}\left(1+\mu^{j} / \mu^{i}\right)-\lambda^{j}\left(1+\mu^{i} / \mu^{j}\right)}{q\left(\mu^{j}-\mu^{i}\right)+b\left(\lambda^{i}-\lambda^{j}\right)+\gamma^{i}-\gamma^{j}} \partial_{i} b \partial_{j} b .
\end{gathered}
$$

Solving equations (31) for $\beta^{i}, \lambda^{i}, \mu^{i}, \gamma^{i}$ and $b$, determining $\eta^{i}$ from the dispersion relation (30) and calculating $p, r, q, a$ from the equations (29) (which are automatically compatible by virtue of (31)), one obtains the general $n$-component reduction of the equation (27). The commutativity conditions will be satisfied identically. We have checked that the system (31) is in involution and its general solution depends, up to reparametrizations $R^{i} \rightarrow \varphi^{i}\left(R^{i}\right)$, on $4 n$ arbitrary functions of one variable. 


\section{Multi-dimensional linearly degenerate systems of hydrodynamic type}

In the recent publication [13], we gave a complete characterization of two-component $(2+1)$-dimensional integrable systems of hydrodynamic type,

$$
\left(\begin{array}{c}
v \\
w
\end{array}\right)_{t}+A(v, w)\left(\begin{array}{c}
v \\
w
\end{array}\right)_{x}+B(v, w)\left(\begin{array}{c}
v \\
w
\end{array}\right)_{y}=0
$$

which possess infinitely many hydrodynamic reductions. The integrability conditions constitute a complicated overdetermined system of second order PDEs for $2 \times 2$ matrices $A$ and $B$. In the particular case when the matrix $A$ is assumed to be linearly degenerate,

$$
A=\left(\begin{array}{cc}
w & 0 \\
0 & v
\end{array}\right)
$$

these conditions imply

$$
B=\left(\begin{array}{cc}
\frac{f(w)}{w-v}-\alpha w^{2} & \frac{f(v)}{w-v} \\
\frac{f(w)}{v-w} & \frac{f(v)}{v-w}-\alpha v^{2}
\end{array}\right)
$$

where $f$ is a cubic polynomial, $f(v)=\alpha v^{3}+\beta v^{2}+\gamma v+\delta$, and $\alpha, \beta, \gamma, \delta$ are arbitrary constants. A remarkable property of this example is that any matrix in the linear pencil $B+\mu A$ is also linearly degenerate (that is, reduces to the diagonal form (32) after an appropriate change of dependent variables). Explicitly, one has

$$
\begin{gathered}
B=\delta B_{1}+\gamma B_{2}+\beta B_{3}+\alpha B_{4}= \\
\delta\left(\begin{array}{ll}
\frac{1}{w-v} & \frac{1}{w-v} \\
\frac{1}{v-w} & \frac{1}{v-w}
\end{array}\right)+\gamma\left(\begin{array}{cc}
\frac{w}{w-v} & \frac{v}{w-v} \\
\frac{w}{v-w} & \frac{v}{v-w}
\end{array}\right)+\beta\left(\begin{array}{cc}
\frac{w^{2}}{w-v} & \frac{v^{2}}{w-v} \\
\frac{w^{2}}{v-w} & \frac{v^{2}}{v-w}
\end{array}\right)+\alpha\left(\begin{array}{cc}
\frac{v w^{2}}{w-v} & \frac{v^{3}}{w-v} \\
\frac{w^{3}}{v-w} & \frac{w v^{2}}{v-w}
\end{array}\right) .
\end{gathered}
$$

Let us introduce the $(5+1)$-dimensional system

$$
\left(\begin{array}{c}
v \\
w
\end{array}\right)_{t}+A\left(\begin{array}{c}
v \\
w
\end{array}\right)_{x}+B_{1}\left(\begin{array}{c}
v \\
w
\end{array}\right)_{y}+B_{2}\left(\begin{array}{c}
v \\
w
\end{array}\right)_{z}+B_{3}\left(\begin{array}{c}
v \\
w
\end{array}\right)_{s}+B_{4}\left(\begin{array}{c}
v \\
w
\end{array}\right)_{r}=0 .
$$

Notice that an arbitrary linear combination of matrices $A$ and $B_{1}, B_{2}, B_{3}, B_{4}$ is linearly degenerate! In the new variables $m=v+w, n=v w$, this systems reduces to

$$
m_{t}+n_{x}+n m_{r}-m n_{r}=0, \quad n_{t}+m n_{x}-n m_{x}+m_{y}+n_{z}+m n_{s}-n m_{s}=0,
$$

taking a fully symmetric form

$$
m_{\tilde{t}}=n_{\tilde{x}}+n m_{\tilde{r}}-m n_{\tilde{r}}, \quad n_{\tilde{z}}=m_{\tilde{y}}+m n_{\tilde{s}}-n m_{\tilde{s}},
$$


after the obvious linear change of independent variables. In the limit $\tilde{s}=\tilde{x}, m_{\tilde{r}}=n_{\tilde{r}}=0$ it reduces to the system (26) from Example 4. Notice that the system (33) arises as the condition of commutativity of two vector fields,

$$
\left[\partial_{\tilde{z}}-m \partial_{\tilde{s}}-\lambda \partial_{\tilde{x}}+\lambda m \partial_{\tilde{r}}, \quad \partial_{\tilde{y}}-n \partial_{\tilde{s}}-\lambda \partial_{\tilde{t}}+\lambda n \partial_{\tilde{r}}\right]=0 .
$$

Let us demonstrate that the system (33) possesses 'enough' hydrodynamic reductions and, therefore, should be regarded as an integrable systems in $5+1$ dimensions. Looking for reductions in the form $m=m\left(R^{1}, \ldots, R^{n}\right), n=n\left(R^{1}, \ldots, R^{n}\right)$ where the Riemann invariants $R^{i}$ solve five commuting systems

$R_{\tilde{t}}^{i}=\lambda^{i}(R) R_{\tilde{x}}^{i}, \quad R_{\tilde{y}}^{i}=\mu^{i}(R) R_{\tilde{x}}^{i}, \quad R_{\tilde{z}}^{i}=\eta^{i}(R) R_{\tilde{x}}^{i}, \quad R_{\tilde{r}}^{i}=\beta^{i}(R) R_{\tilde{x}}^{i}, \quad R_{\tilde{s}}^{i}=\gamma^{i}(R) R_{\tilde{x}}^{i}$,

and substituting into (33), we obtain

$$
\left(\lambda^{i}-n \beta^{i}\right) \partial_{i} m=\left(1-m \beta^{i}\right) \partial_{i} n, \quad\left(\eta^{i}-m \gamma^{i}\right) \partial_{i} n=\left(\mu^{i}-n \gamma^{i}\right) \partial_{i} m .
$$

Setting $\partial_{i} n=\varphi^{i} \partial_{i} m$, one obtains expressions for $\lambda^{i}$ and $\mu^{i}$ in the form

$$
\lambda^{i}=n \beta^{i}+\left(1-m \beta^{i}\right) \varphi^{i}, \quad \mu^{i}=n \gamma^{i}+\left(\eta^{i}-m \gamma^{i}\right) \varphi^{i},
$$

as well as the consistency condition

$$
\partial_{i} \partial_{j} m=\frac{\partial_{j} \varphi^{i}}{\varphi^{j}-\varphi^{i}} \partial_{i} m+\frac{\partial_{i} \varphi^{j}}{\varphi^{i}-\varphi^{j}} \partial_{j} m .
$$

Inserting the expressions for $\lambda^{i}$ and $\mu^{i}$ into the commutativity conditions

$$
\frac{\partial_{j} \lambda^{i}}{\lambda^{j}-\lambda^{i}}=\frac{\partial_{j} \mu^{i}}{\mu^{j}-\mu^{i}}=\frac{\partial_{j} \eta^{i}}{\eta^{j}-\eta^{i}}=\frac{\partial_{j} \beta^{i}}{\beta^{j}-\beta^{i}}=\frac{\partial_{j} \gamma^{i}}{\gamma^{j}-\gamma^{i}}
$$

one ends up with the following equations for $\eta^{i}, \beta^{i}, \gamma^{i}, \varphi^{i}$ and $m$ :

$$
\begin{gathered}
\frac{\partial_{j} \eta^{i}}{\eta^{j}-\eta^{i}}=\frac{\partial_{j} \beta^{i}}{\beta^{j}-\beta^{i}}=\frac{\partial_{j} \gamma^{i}}{\gamma^{j}-\gamma^{i}}=\frac{\beta^{i} \eta^{i}-\gamma^{i}}{\left(1-m \beta^{j}\right)\left(\eta^{i}-m \gamma^{i}\right)-\left(1-m \beta^{i}\right)\left(\eta^{j}-m \gamma^{j}\right)} \partial_{j} m \\
\frac{\partial_{j} \varphi^{i}}{\varphi^{j}-\varphi^{i}}=\frac{\beta^{i} \eta^{j}-\gamma^{i}+m\left(\gamma^{i} \beta^{j}-\gamma^{j} \beta^{i}\right)}{\left(1-m \beta^{j}\right)\left(\eta^{i}-m \gamma^{i}\right)-\left(1-m \beta^{i}\right)\left(\eta^{j}-m \gamma^{j}\right)} \partial_{j} m \\
\partial_{i} \partial_{j} m=\frac{\gamma^{j}-\gamma^{i}+\beta^{i} \eta^{j}-\beta^{j} \eta^{i}+2 m\left(\gamma^{i} \beta^{j}-\gamma^{j} \beta^{i}\right)}{\left(1-m \beta^{j}\right)\left(\eta^{i}-m \gamma^{i}\right)-\left(1-m \beta^{i}\right)\left(\eta^{j}-m \gamma^{j}\right)} \partial_{i} m \partial_{j} m .
\end{gathered}
$$

It has been verified directly that this system is in involution and its general solution depends, modulo reparametrizations $R^{i} \rightarrow f^{i}\left(R^{i}\right)$, on $4 n$ arbitrary functions of one variable, thus manifesting the integrability of the $(5+1)$-dimensional system $(33)$. 


\section{Appendix}

Here we apply the method of hydrodynamic reductions to the classification of integrable PDEs of the form

$$
\theta_{t x}+\theta_{z y}=f\left(\theta_{x x}, \theta_{x y}, \theta_{y y}\right) .
$$

Introducing new variables $\theta_{x x}=u, \theta_{x y}=v, \theta_{y y}=w, \theta_{t x}=p, \theta_{z y}=f(u, v, w)-p$, one rewrites (35) in the quasilinear form

$$
\begin{gathered}
u_{y}=v_{x}, \quad u_{t}=p_{x}, \quad v_{y}=w_{x}, \quad v_{t}=p_{y}, \\
v_{z}=(f(u, v, w)-p)_{x}, \quad w_{z}=(f(u, v, w)-p)_{y} .
\end{gathered}
$$

Hydrodynamic reductions are sought in the form $u=u\left(R^{1}, \ldots, R^{n}\right), v=v\left(R^{1}, \ldots, R^{n}\right)$, $w=w\left(R^{1}, \ldots, R^{n}\right), p=p\left(R^{1}, \ldots, R^{n}\right)$ where the Riemann invariants $R^{1}, \ldots, R^{n}$ solve a triple of commuting hydrodynamic type systems (2). The substitution into (36) implies

$$
\partial_{i} p=\lambda^{i} \partial_{i} u, \quad \partial_{i} v=\mu^{i} \partial_{i} u, \quad \partial_{i} w=\left(\mu^{i}\right)^{2} \partial_{i} u,
$$

along with the dispersion relation

$$
\lambda^{i}=f_{u}+f_{v} \mu^{i}+f_{w}\left(\mu^{i}\right)^{2}-\mu^{i} \eta^{i} .
$$

Substituting $\lambda^{i}$ into the commutativity conditions (3), and taking into account that the compatibility conditions for the relations (37) imply

$$
\partial_{i} \partial_{j} u=\frac{\partial_{j} \mu^{i}}{\mu^{j}-\mu^{i}} \partial_{i} u+\frac{\partial_{i} \mu^{j}}{\mu^{i}-\mu^{j}} \partial_{j} u,
$$

one arrives at the following system:

$$
\begin{gathered}
\partial_{j} \mu^{i}=\frac{S_{i j}}{f_{w}\left(\mu^{j}-\mu^{i}\right)+\eta^{i}-\eta^{j}} \partial_{j} u, \quad \partial_{j} \eta^{i}=\frac{\eta^{j}-\eta^{i}}{\mu^{j}-\mu^{i}} \frac{S_{i j}}{f_{w}\left(\mu^{j}-\mu^{i}\right)+\eta^{i}-\eta^{j}} \partial_{j} u, \\
\partial_{i} \partial_{j} u=\frac{2}{\mu^{j}-\mu^{i}} \frac{S_{i j}}{f_{w}\left(\mu^{j}-\mu^{i}\right)+\eta^{i}-\eta^{j}} \partial_{i} u \partial_{j} u ;
\end{gathered}
$$

here

$$
\begin{gathered}
S_{i j}=f_{u u}+\left(\mu^{i}+\mu^{j}\right) f_{u v}+\left(\left(\mu^{i}\right)^{2}+\left(\mu^{j}\right)^{2}\right) f_{u w}+\mu^{i} \mu^{j} f_{v v}+ \\
\mu^{i} \mu^{j}\left(\mu^{i}+\mu^{j}\right) f_{v w}+\left(\mu^{i}\right)^{2}\left(\mu^{j}\right)^{2} f_{w w} .
\end{gathered}
$$

Compatibility conditions for the system (39) are of the form

$$
\begin{gathered}
\partial_{k}\left(\partial_{j} \mu^{i}\right)-\partial_{j}\left(\partial_{k} \mu^{i}\right)=(\ldots) \partial_{j} u \partial_{k} u, \quad \partial_{k}\left(\partial_{j} \eta^{i}\right)-\partial_{j}\left(\partial_{k} \eta^{i}\right)=(\ldots) \partial_{j} u \partial_{k} u, \\
\partial_{k}\left(\partial_{j} \partial_{i} u\right)-\partial_{j}\left(\partial_{k} \partial_{i} u\right)=(\ldots) \partial_{j} u \partial_{k} u
\end{gathered}
$$

where dots (...) denote complicated rational expressions in $\mu^{i}, \mu^{j}, \mu^{k}$ and $\eta^{i}, \eta^{j}, \eta^{k}$ whose coefficients are functions of the derivatives of $f$ up to third order. Equating these rational expressions to zero one arrives at the following system for $f$ :

$$
f_{u u}=f_{w w}=f_{u v}=f_{w v}=f_{v v}-2 f_{u w}=f_{v v v}=0 .
$$

Up to elementary changes of variables, the general nonlinear solution of this system corresponds to the second heavenly equation (13). 


\section{Concluding remarks}

We have demonstrated that the requirement of existence of 'sufficiently many' $n$-component reductions can be used as the effective criterion providing the test for integrability of multi-dimensional dispersionless PDEs. We believe that using the approach outlined in this paper along with the available computer algebra packages (we have used Mathematica 5.0 ), one can obtain complete lists of multi-dimensional integrable systems within various particularly interesting classes, hyperbolic systems of hydrodynamic type being one of them. Partial classification results can be found in $[12,13,28]$. The main problems arising here are the complexity of integrability conditions (making difficult their geometric analysis), and the volume of symbolic calculations required.

\section{Acknowledgements}

We would like to thank M. Dunajski and M. Pavlov for stimulating discussions and references.

\section{References}

[1] V.E. Adler, A.I. Bobenko and Yu.B. Suris, Classification of integrable equations on quad-graphs. The consistency approach, Comm. Math. Phys. 233, N3 (2003) 513543.

[2] B. Banos, On symplectic classification of effective 3-forms and Monge-Ampére equations, Diff. Geom. Appl. 19 (2003) 147-166.

[3] A. Boyarsky, A. Marshakov, O. Ruchayskiy, P. Wiegmann and A. Zabrodin, Associativity equations in dispersionless integrable hierarchies. Phys. Lett. B 515, N3-4 (2001) 483-492.

[4] M. Burnat, The method of characteristics and Riemann's invariants for multidimensional hyperbolic systems. (Russian) Sibirsk. Mat. Z. 11 (1970) 279-309.

[5] R. Carroll and Y. Kodama, Characterization and solution of the dispersionless Hirota equations. Nonlinear physics: theory and experiment (Lecce, 1995), 53-59, World Sci. Publishing, River Edge, NJ, 1996.

[6] M. Dunajski, The nonlinear graviton as an integrable system, DPhil thesis, Oxford, 1998.

[7] M. Dunajski, L.J. Mason and N.M.J. Woodhouse, From 2D integrable systems to self-dual gravity, J. Phys. A: Math. Gen. 31 (1998) 6019-6028.

[8] M. Dunajski, L.J. Mason and K.P. Tod, Einstein-Weyl geometry, the dKP equation and twistor theory, J. Geom. Phys. 37 (2001) 63-92. 
[9] M. Dunajski, A class of Einstein-Weyl spaces associated to an integrable system of hydrodynamic type, arXiv:nlin.SI/0311024.

[10] E.V. Ferapontov, D. A. Korotkin and V.A. Shramchenko, Boyer-Finley equation and systems of hydrodynamic type, Class. Quantum Grav. 19 (2002) L1-L6.

[11] E.V. Ferapontov and M.V. Pavlov, Hydrodynamic reductions of the heavenly equation, Class. Quantum Grav. 20 (2003) 2429-2441.

[12] E.V. Ferapontov and K.R. Khusnutdinova, On integrability of (2+1)-dimensional quasilinear systems, to appear in Comm. Math. Phys., nlin.SI/0305044.

[13] E.V. Ferapontov and K.R. Khusnutdinova, The characterization of two-component $(2+1)$-dimensional integrable systems of hydrodynamic type, nlin.SI/0310021.

[14] J. Gibbons and Y. Kodama, A method for solving the dispersionless KP hierarchy and its exact solutions. II. Phys. Lett. A 135, N3 (1989) 167-170.

[15] J. Gibbons and S. P. Tsarev, Reductions of the Benney equations, Phys. Lett. A 211 (1996) 19-24.

[16] J. Gibbons and S. P. Tsarev, Conformal maps and reductions of the Benney equations, Phys. Lett. A 258 (1999) 263-271.

[17] J.D.E. Grant, On self-dual gravity, Phys. Rev. D 47 (1993) 2606-2612.

[18] F. Guil, M. Manas and L. Martinez Alonso, On the Whitham Hierarchies: Reductions and Hodograph Solutions, nlin.SI/0209051.

[19] J. Hietarinta, Equations that pass Hirota's three-soliton condition and other tests of integrability. Nonlinear evolution equations and dynamical systems (Kolymbari, 1989), 46-50, Res. Rep. Phys., Springer, Berlin, 1990.

[20] I.M. Krichever, The averaging method for two-dimensional "integrable" equations, Funct. Anal. Appl. 22, no. 3 (1988) 200-213.

[21] I.M. Krichever, Spectral theory of two-dimensional periodic operators and its applications, Russian Math. Surveys 44, no. 2 (1989) 145-225.

[22] I.M. Krichever, A. Marshakov and A. Zabrodin, Integrable structure of the Dirichlet boundary problem in multi-connected domains, arXiv:hep-th/0309010.

[23] V.V. Lychagin, V.N. Rubtsov and I.V. Chekalov, A classification of Monge-Ampre equations, Ann. Sci. cole Norm. Sup. (4) 26 (1993) 281-308.

[24] M. Manas, L. Martinez Alonso and E. Medina, Reductions and hodograph solutions of the dispersionless KP hierarchy, J. Phys. A: Math. Gen. 35 (2002) 401-417.

[25] M. Manas and L. Martinez Alonso, A hodograph transformation which applies to the heavenly equation, nlin.SI/0209050. 
[26] L. Martnez Alonso, A.B. Shabat, Towards a theory of differential constraints of a hydrodynamic hierarchy, J. Nonlinear Math. Phys. 10, N2 (2003) 229-242.

[27] M.V. Pavlov, Integrable hydrodynamic chains, J. Math. Phys. 44 , N9 (2003) 4134; nlin.SI/0301010.

[28] M.V. Pavlov, Classification of the integrable Egorov hydrodynamic chains, to appear in Theor. and Math. Phys. (2003).

[29] Z. Peradzyński, Nonlinear plane $k$-waves and Riemann invariants. Bull. Acad. Polon. Sci. Sr. Sci. Tech. 19 (1971) 625-632.

[30] J.F. Plebański, Some solutions of complex Einstein equations, J. Math. Phys. 16 (1975) 2395-2402.

[31] J.F. Plebański and M. Przanowski, The Lagrangian for a self-dual gravitational field as a limit of the SDYM Lagrangian, Phys. lett. A 212 (1996) 22-28.

[32] S.P. Tsarev, Geometry of Hamiltonian systems of hydrodynamic type. Generalized hodograph method. Izvestija AN USSR Math. 54, N5 (1990) 1048-1068.

[33] E.V. Zakharov, Dispersionless limit of integrable systems in $2+1$ dimensions, in Singular Limits of Dispersive Waves, Ed. N.M. Ercolani et al., Plenum Press, NY, (1994) 165-174. 